\title{
Clinical characteristics of the first cases of
} invasive candidiasis in China due to panechinocandin-resistant Candida tropicalis and Candida glabrata isolates with delineation of their resistance mechanisms

This article was published in the following Dove Press journal: Infection and Drug Resistance

Meng Xiao, ${ }^{1,2, *}$ Xin Fan, ${ }^{1-3, *}$ Xin Hou, ${ }^{1,2,4, *}$ Sharon CA Chen, ${ }^{5}$ He Wang, ${ }^{1,2}$ Fanrong Kong, ${ }^{5} \mathrm{Zi}$-Yong Sun, ${ }^{6, *}$ YunZhuo Chu, ${ }^{7}$ Ying-Chun $\mathrm{Xu},{ }^{1,2}$

'Department of Clinical Laboratory, Peking Union Medical College Hospital, Chinese Academy of Medical Sciences, Beijing, People's Republic of China; ${ }^{2}$ Beijing Key Laboratory for Mechanisms Research and Precision Diagnosis of Invasive Fungal Diseases, Beijing, People's Republic of China; ${ }^{3}$ Department of Infectious Diseases and Clinical Microbiology, Beijing Chaoyang Hospital, Beijing, People's Republic of China; ${ }^{4}$ Graduate School, Peking Union Medical College, Chinese Academy of Medical Sciences, Beijing, People's Republic of China; ${ }^{5}$ Center for Infectious Diseases and Microbiology Laboratory Services, ICPMR, New South Wales Health Pathology, Westmead Hospital, The University of Sydney, NSW, Australia; 'Department of Clinical Laboratory, Tongji Hospital, Huazhong University of Science and Technology, Wuhan, People's Republic of China; ${ }^{7}$ Department of Clinical Laboratory, The First Affiliated Hospital of Chinese Medical University, Shenyang, People's Republic of China

*These authors contributed equally to this work

Correspondence: Ying-Chun Xu Department of Clinical Laboratory, Peking Union Medical College Hospital, Chinese Academy of Medical Sciences, Shuaifuyuan Street, Beijing 100730, People's Republic of China

Tel +861069159766

Email xycpumch@।39.com

Yun-Zhuo Chu

Department of Clinical Laboratory, The First Affiliated Hospital of Chinese Medical University, No. I 55 North Nanjing Road, Shenyang I I000I,

People's Republic of China

Tel +862483282167

Email cyz6630@163.com

\begin{abstract}
Echinocandin antifungal agents have become the first-line therapy for invasive candidiasis (IC) in many countries. Despite their increasing use, resistance to this class of drug is, overall, still uncommon. Here, we report two patients from the People's Republic of China with IC, one with infection caused by pan-echinocandin-resistant Candida tropicalis and the other by pan-echinocandin-resistant Candida glabrata. We also describe the mechanisms of drug resistance of these isolates. The echinocandin-resistant $C$. glabrata isolate was cultured from ascitic fluid of a 46-year-old male patient with intra-abdominal IC developing after surgery in 2012. This patient had had no prior antifungal exposure. The echinocandin-resistant C. tropicalis isolate was cultured from chest drainage fluid of a 60 -year-old female patient with severe coronary disease and lung infection. Prior to culture and identification of the isolate, the patient had received micafungin treatment for 19 days. Both isolates were cross-resistant to micafungin, anidulafungin, and caspofungin, with minimum inhibitory concentration values of $\geq 2 \mu \mathrm{g} / \mathrm{mL}$. The amino acid substitution E655K was found adjacent to the FKS2 HS1 region of the C. glabrata isolate, while the substitution S80P were found in the FKS1 HS1 region of the $C$. tropicalis isolate. This report highlights the emergence of echinocandin resistance in two important non-albicans Candida species. Although the overall prevalence of echinocandin resistance is low in the People's Republic of China, monitoring of antifungal susceptibility trends in all Candida species is warranted.
\end{abstract}

Keywords: Candida tropicalis, Candida glabrata, echinocandins, antifungal resistance, People's Republic of China

\section{Introduction}

Invasive candidiasis (IC) is increasing in prevalence, especially among immunocompromised patients and those with serious underlying disease. In addition, mortality from IC remains high. ${ }^{1}$ Candida tropicalis and Candida glabrata are two major pathogenic nonalbicans Candida species. C. glabrata is the second most common cause of candidemia in Europe and America, while C. tropicalis has become the first and second leading cause of IC in India and the People's Republic of China, respectively. ${ }^{1-3}$ These species are notable for their resistance or reduced susceptibility to azole antifungal agents. ${ }^{4,5}$

As such, the echinocandins are increasingly used as first-line therapy for IC, because of their good efficacy and safety profiles. ${ }^{4,6}$ However, reduced susceptibility 
and resistance among Candida species to these agents has also been noted, linked to mutations within specific hotspot (HS) regions of the Candida FKS genes. ${ }^{7,8}$ The emergence of echinocandin resistance has been most concerning for $C$. glabrata, especially in North America, while echinocandin resistance in other Candida species and in other geographical regions remains more uncommon. ${ }^{6,9}$

In the China Hospital Invasive Fungal Surveillance Net (CHIF-NET) 2013 program, one C. tropicalis and C. glabrata isolate each was found to be resistant to all three licensed echinocandin agents, ${ }^{3,10}$ being the first pan-echinocandinresistant isolates identified in the country. Here, we report the clinical features of the patients affected by these panechinocandin-resistant strains. We also determined the mechanism of resistance in the isolates.

\section{Case presentation \\ Case I: Infection with echinocandin- resistant C. glabrata}

On September 27, 2012, a 46-year-old male was admitted to the Department of Pancreatic Surgery in a hospital in the Northeast region of the People's Republic of China with fever and an abdominal wound infection (Table 1). He had experienced a right hepatectomy, subtotal gastrectomy, transverse colon loop ostomy, and right thoracic cavity drainage and closure 38 days prior in another hospital owning to severe trauma. He was immunocompetent. Before admission, the patient had received imipenem therapy for 10 days because of Escherichia coli bacteremia, but there was no history of exposure to antifungal agents.

Table I Clinical features of two patients with echinocandin-resistant candidiasis and in vitro susceptibilities of two isolates

\begin{tabular}{|c|c|c|}
\hline Characteristics & Candida glabrata patient & Candida tropicalis patient \\
\hline Age (years) & 46 & 69 \\
\hline Gender & Male & Female \\
\hline Date of admission & September 27, 2012 & May 10,2013 \\
\hline Department of admission & Department of Pancreatic Surgery & ICU \\
\hline Reason for hospital admission & Fever and abdominal incision infection & Asthma, pulmonary infection \\
\hline Underlying disease & $\begin{array}{l}\text { Right hepatectomy, subtotal gastrectomy, } \\
\text { transverse colon loop ostomy, right closed } \\
\text { chest drainage }\end{array}$ & $\begin{array}{l}\text { Coronary heart disease, cardiac valve } \\
\text { replacement, multiple organ dysfunction } \\
\text { syndrome }\end{array}$ \\
\hline CHIF-NET strain no. & $12 Z 1 \mid 32$ & I3TJ350 \\
\hline Site of isolation & Ascitic fluid & Left chest drainage \\
\hline Date of isolation & October I, 2012 & July 18,2013 \\
\hline \multicolumn{3}{|c|}{ Clinical status at time of positive culture } \\
\hline Immunosuppressive state & No & No \\
\hline Neutropenia(<109/L) & No & No \\
\hline Presence of CVC & No & No \\
\hline Broad-spectrum antibiotics & Yes & Yes \\
\hline Total parenteral nutrition & No & Yes \\
\hline Mechanical ventilation & No & Yes \\
\hline Surgery within 30 days & Yes & Yes \\
\hline Previous antifungal agents within 30 days & No & Micafungin, 18 days \\
\hline Indwelling urinary catheter & No & Yes \\
\hline \multicolumn{3}{|l|}{ Therapy } \\
\hline Antifungal CVC removal & Not applicable & No \\
\hline Antifungal after culture & Fluconazole, $200 \mathrm{mg} / \mathrm{d}$ & Voriconazole, $200 \mathrm{mg} / \mathrm{d}$ \\
\hline Outcome & Recovered & Dead \\
\hline \multicolumn{3}{|c|}{ Antifungal susceptibilities (MIC [mg/L]/category) } \\
\hline Micafungin & $\geq 8 / R$ & $2 / R$ \\
\hline Anidulafungin & $\geq 8 / R$ & $2 / R$ \\
\hline Caspofungin & $\geq 8 / R$ & $4 / R$ \\
\hline Fluconazole & 32/SDD & $2 / S$ \\
\hline Voriconazole & $\mathrm{I} / \mathrm{WT}$ & $0.125 / \mathrm{S}$ \\
\hline Itraconazole & $\mathrm{I} / \mathrm{WT}$ & $0.25 / \mathrm{S}$ \\
\hline Posaconazole & $2 / \mathrm{WT}$ & $0.25 / \mathrm{WT}$ \\
\hline Amphotericin B & $0.5 / \mathrm{WT}$ & $0.5 / \mathrm{WT}$ \\
\hline 5-Flucytosine & $\leq 0.06 / \mathrm{WT}$ & $\leq 0.06 / \mathrm{WT}$ \\
\hline
\end{tabular}

Abbreviations: ICU, intensive care unit; CHIF-NET, China Hospital Invasive Fungal Surveillance Net; CVC, central venous catheter; MIC, minimum inhibitory concentration; S, susceptible; SDD, susceptible dose-dependent; R, resistant; WT, wild-type. 
On day 4 of admission, the local laboratory reported growth of C. glabrata in the ascitic fluid of the patient collected on day 2 ; however, antifungal susceptibility testing was not performed. Fluconazole therapy $200 \mathrm{mg} / \mathrm{d}$ was initiated immediately on day 4 for 23 days duration. No other cultures were positive for fungi. The patient made good clinical recovery and was discharged on October 24 (day 27).

\section{Case 2: Echinocandin-resistant C. tropicalis} On May 10, 2013, a 69-year-old female was admitted to the intensive care unit of a hospital in the Middle region of the People's Republic of China with severe asthma and pulmonary infection (Table 1). She suffered from severe coronary disease, underwent aortic valve replacement 1 month prior in another hospital, and had since developed multiple organ dysfunction. A previous sputum culture within the last 30 days was positive for Acinetobacter baumannii and Candida albicans. From day 1 of admission, she received meropenem $3 \mathrm{~g} / \mathrm{d}$ for 22 days and micafungin $100 \mathrm{mg} / \mathrm{d}$ for 18 days.

On day 8 of admission, a chest tube was placed in the left chest wall for drainage of a pleural effusion from unresolved pneumonia. On day 33, C. tropicalis was cultured from the pleural drainage fluid. The local laboratory performed antifungal susceptibility testing of the isolate by the disk diffusion method, ${ }^{11}$ but only for fluconazole, and reported a "susceptible" result. Voriconazole therapy $(200 \mathrm{mg} / \mathrm{d})$ was initiated and continued till day 99 of admission when the patient passed away from heart failure.

\section{Materials and methods Ethics statement}

Written informed consent to publish their case details was obtained from the patient, or next of kin where the patient was unable to consent. The Human Research Ethics Committee of Peking Union Medical College Hospital has provided permission to publish this report (S-628).

\section{Detection of the pan-echinocandin- resistant Candida isolates from CHIF- NET national surveillance}

CHIF-NET was established as a nationwide surveillance program in the People's Republic of China to monitor the trends in the epidemiology of invasive yeast infections and to provide up-to-date susceptibility data on antifungal agents. ${ }^{2,3,10} \mathrm{CHIF}$ NET 2013 comprised the fourth consecutive surveillance year of the program, held from August 1, 2012 to July 31, 2013. Generally, all yeast isolates collected in CHIF-NET 2013 were forwarded to the central laboratory, the Department of Clinical Laboratory, Peking Union Medical College Hospital. Confirmation of species identification was carried out by an algorithm of matrix-assisted laser desorption/ionizationtime of flight mass spectrometry (Vitek MS; bioMérieux, Marcy l'Etoile, France) supplemented with rDNA internal transcribed spacer (ITS) sequencing. ${ }^{12}$ The in vitro susceptibility to nine antifungal drugs, including three echinocandins (caspofungin, micafungin, and anidulafungin), amphotericin $\mathrm{B}$, and 5-flucytosine, was determined using Sensititre YeastOne $^{\mathrm{TM}}$ YO10 methodology (Sensititre; Thermo Scientific, Cleveland, OH, USA) following the manufacturer's instructions. Current available species-specific clinical breakpoint or epidemiological cut-off values were used for interpretation of results (Table S1). ${ }^{13-15}$ Only one C. glabrata (CHIF-NET study no. 12Z1132) and one C. tropicalis (study no. 13TJ350) isolate, which were isolated from the patients described above, were resistant to all the echinocandins (Table 1).

\section{Analysis of the FKS gene}

For C. glabrata strain 12Z1132, sequencing of FKS1 and FKS2 genes was carried out as described by Zimbeck et al; ${ }^{8}$ sequences of C. glabrata strain CBS 138 (GenBank accession numbers XM_446406 and XM_448401 for FKS1 and $F K S 2$, respectively) were the wild-type (WT) reference sequences. For C. tropicalis strain 13TJ350, sequencing of FKS1 gene was carried out as described by Jensen et al, ${ }^{7}$ with the sequence of $C$. tropicalis ATCC 750 (GenBank accession number EU676168) representing the WT reference sequence.

\section{Nucleotide sequences}

The partial FKS2 gene sequence of C. glabrata $12 \mathrm{Z} 1132$ and partial FKS1 gene sequence of $C$. tropicalis strain 13TJ350 have been deposited in GenBank database, with GenBank accession numbers of MF667536 and MF667537, respectively.

\section{Results}

\section{Echinocandin-resistant isolates}

In CHIF-NET 2013, 2,687 yeast isolates were collected from 48 hospitals in the People's Republic of China where C. tropicalis (413 isolates, 15.4\%) and C. glabrata sensu stricto (254 isolates, $9.5 \%$ ) were the third and fourth most common species. However, only C. tropicalis isolate $13 \mathrm{TJ} 350$ and C. glabrata isolate $12 Z 1132$ were echinocandin-resistant. These were the first pan-echinocandin-resistant $C$. tropicalis and C. glabrata isolates identified in the People's Republic of China. ${ }^{3,10}$ 


\section{Antifungal susceptibilities}

C. glabrata strain $12 \mathrm{Z1132}$ was resistant to all three echinocandin agents, with minimum inhibitory concentrations of $\geq 8 \mu \mathrm{g} / \mathrm{mL}$ (Table 1). C. tropicalis strain 13TJ350 was also pan-echinocandin resistant, with minimum inhibitory concentrations of $4 \mu \mathrm{g} / \mathrm{mL}, 2 \mu \mathrm{g} / \mathrm{mL}$, and $2 \mu \mathrm{g} / \mathrm{mL}$ to caspofungin, micafungin, and anidulafungin, respectively (Table 1). Both strains were susceptible or of WT phenotype to 5-flucytosine, amphotericin B, and four azoles tested, except for C. glabrata strain 12Z1132 which was susceptible in a dose-dependent manner to fluconazole (Table 1).

\section{Analysis of FKS genes}

A mutation leading to the amino acid substitution S80P was found in HS1 region of the FKS1 gene of $C$. tropicalis strain 13TJ350. However, no amino acid substitution was detected in HS regions of FKS1 and FKS2 genes in C. glabrata strain 12Z1132. Instead, there was a mutation leading to the amino acid substitution E655K adjacent to HS1 region of the FKS2 gene.

\section{Discussion}

Because there are only a few classes of antifungal agents, for example, the azoles, echinocandins and polyenes, in clinical use, options for antifungal therapy are relatively limited. Facing the challenges posed by azole resistance, echinocandins have become the preferred therapy for IC. ${ }^{4,6}$ Overall, resistance to the echinocandins appears to be uncommon. From a recent global surveillance report, echinocandin susceptibility rates were over $95 \%$ for C. albicans as well as for the more commonly encountered non-albicans Candida species. ${ }^{16}$ However, since the first report of reduced susceptibility to caspofungin in C. albicans in $2004,{ }^{17}$ echinocandin resistance among Candida isolates has been increasingly identified among different Candida species, raising global concern. ${ }^{4,5,16}$

Of the three licensed echinocandin agents, caspofungin and micafungin were approved for marketing by the China Food and Drug Administration in 2006, while no commercial brand of anidulafungin drug has been approved for use in the People's Republic of China, until now. Although the use of echinocandin drugs has increased since 2006, the prevalence of echinocandin-resistant strains is not known. At the inception of the first CHIF-NET study (2009), no clinical laboratory was routinely performing antifungal susceptibility testing for echinocandins, underscoring lack of data for this important phenomenon.

Specifically, at the initial stage of the CHIF-NET study, the focus of surveillance involved drug susceptibility only to fluconazole and voriconazole and by using diffusion methods. ${ }^{2}$ To overcome the lack of data on echinocandin susceptibility, in 2015, the CHIF-NET study group initiated surveillance of echinocandin susceptibilities on common non-albicans Candida species. In the 3-year review, only one C. glabrata strain resistant to anidulafungin $(0.4 \%)$ was detected, while no strain was found to be resistant to caspofungin or micafungin. ${ }^{2}$ Since then, the first panechinocandin-resistant Candida strains in the People's Republic of China have been identified by Fan et $\mathrm{al}^{3}$ and Hou et $\mathrm{al}^{10}$ in the 5 -year review of C. glabrata and C. tropicalis species, respectively.

Of note, most echinocandin resistance in Candida species worldwide have been documented in C. glabrata $^{4,5,16} \mathrm{In}$ addition, the prevalence of echinocandin resistance has varied with geographic region: echinocandin-resistant $C$. glabrata is more common in North America $(7 \%-10 \%)$ than in other continents $(0 \%-2 \%) .{ }^{16}$ Echinocandin resistance is typically associated with prior antifungal drug exposure. ${ }^{4,9,18}$ However, in our patient with infection due to an echinocandin-resistant C. glabrata, no previous antifungal use was evident. The infection was thought to be acquired after the patient's abdominal operation, supporting the notion that, even without typical risk factors, ${ }^{18}$ patients may become infected by echinocandin-resistant strains.

Echinocandin resistance in C. tropicalis appears to be uncommon $(<1 \%) \cdot{ }^{3,6,14,16}$ Reports of infections caused by such strains have included three cases of breakthrough candidemia in allogeneic stem cell recipients, ${ }^{19}$ and a case of candidal esophagitis in a patient with acute myelogenous leukemia. ${ }^{20}$ Compared with published reports, our patient (patient 2) had exposure to at least one echinocandin, but the patient was not immunosuppressed.

It is well established that mutations in the FKS gene account for echinocandin resistance and that their presence is linked with failure of echinocandin therapy. ${ }^{4,7,21}$ Although other resistant mechanisms, for example, alterations in the mismatch repair gene $m s h 2$, have also been reported, their role in conferring resistance is less certain. ${ }^{9}$ In C. albicans and $C$. tropicalis, amino acid substitutions associated with resistance have mainly been documented in two HS regions (HS1 and HS2) of the FKS1 gene, while in C. glabrata, these mutations are in homologous regions of FKS1 and FKS2 genes. ${ }^{4,721}$ In the present study, the S80P substitution was found in HS1 region of the FKS1 gene in C. tropicalis strain 13TJ350; this substitution has been linked to echinocandin resistance in C. tropicalis. ${ }^{7}$ Although there was no amino acid substitution in HS regions of the FKS1 and FKS2 genes 
in C. glabrata strain 12Z1132, the E655K substitution was identified upstream to $F K S 2$ gene HS1 region compared with the WT strain. However, as a limitation of the study, the correlation between the novel E655K substitution identified in the C. glabrata isolate and the strain's resistance phenotype remains to be confirmed.

The emergence of multidrug resistant Candida isolates, for example, isolates resistant to both azoles and echinocandins that occurred in C. glabrata, has also been a concern. ${ }^{4,5}$ Fortunately, both our pan-echinocandin-resistant Candida isolates were not resistant to any of the azoles tested. Although the $C$. tropicalis infected patient finally passed away because of poor health, the C. glabrata infected patient exhibited good clinical response to fluconazole treatment. Importantly, since the writing of this report, an additional isolate of C. glabrata resistant to all azoles and all echinocandins has been identified. ${ }^{10}$ This emergence of multi class drug resistance signals the need for increased vigilance by clinicians and scientists alike.

\section{Conclusion}

In conclusion, we describe the clinical presentation of the first patients with IC caused by pan-echinocandin-resistant $C$. tropicalis and C. glabrata isolates. Taking the rapid increase of azole resistance in these two species into account, ${ }^{3,10}$ the emergence of echinocandin resistance will pose challenges for management of patients with IC in the People's Republic of China.

\section{Acknowledgments}

This work was supported by a CAMS Innovation Fund for Medical Sciences (2016-I2M-1-014), a Beijing Innovation Base Cultivation and Development Special Fund (Z171100002217068), a PUMCH Out-standing Young Talents Program (JQ201703), and a National Key Research and Development Program of China (No. 2016YFC0901500). The funders had no role in study design, data collection, and interpretation, or the decision to submit the work for publication.

\section{Author contributions}

All authors contributed toward data analysis, drafting, and critically revising the paper, and agree to be accountable for all aspects of the work.

\section{Disclosure}

The authors report no conflicts of interest in this work.

\section{References}

1. Guinea J. Global trends in the distribution of Candida species causing candidemia. Clin Microbiol Infect. 2014;20(Suppl 6):5-10.

2. Xiao M, Fan X, Chen SC, et al. Antifungal susceptibilities of Candida glabrata species complex, Candida krusei, Candida parapsilosis species complex and Candida tropicalis causing invasive candidiasis in China: 3 year national surveillance. JAntimicrob Chemother. 2015;70(3):802-810.

3. Fan X, Xiao M, Liao K, et al. Notable increasing trend in azole nonsusceptible Candida tropicalis causing invasive candidiasis in China (August 2009 to July 2014): molecular epidemiology and clinical azole consumption. Front Microbiol. 2017;8:464.

4. Perlin DS, Rautemaa-Richardson R, Alastruey-Izquierdo A. The global problem of antifungal resistance: prevalence, mechanisms, and management. Lancet Infect Dis. 2017;17(12):e383-e392.

5. Perlin DS, Shor E, Zhao Y. Update on antifungal drug resistance. Curr Clin Microbiol Rep. 2015;2(2):84-95.

6. Pfaller MA, Rhomberg PR, Messer SA, Jones RN, Castanheira M Isavuconazole, micafungin, and 8 comparator antifungal agents' susceptibility profiles for common and uncommon opportunistic fungi collected in 2013: temporal analysis of antifungal drug resistance using CLSI species-specific clinical breakpoints and proposed epidemiological cutoff values. Diagn Microbiol Infect Dis. 2015;82(4):303-313.

7. Jensen RH, Johansen HK, Arendrup MC. Stepwise development of a homozygous S80P substitution in Fks1p, conferring echinocandin resistance in Candida tropicalis. Antimicrob Agents Chemother. 2013;57(1):614-617.

8. Zimbeck AJ, Iqbal N, Ahlquist AM, et al. FKS mutations and elevated echinocandin MIC values among Candida glabrata isolates from U.S. population-based surveillance. Antimicrob Agents Chemother. 2010;54(12):5042-5047.

9. Delliere S, Healey K, Gits-Muselli M, et al. Fluconazole and echinocandin resistance of Candida glabrata correlates better with antifungal drug exposure rather than with MSH2 mutator genotype in a French cohort of patients harboring low rates of resistance. Front Microbiol. 2016;7:2038.

10. Hou X, Xiao M, Chen SC, et al. Molecular epidemiology and antifungal susceptibility of Candida glabrata in China (August 2009 to July 2014): a multicenter study. Front Microbiol. 2017;8:880.

11. CLSI. M44-A2. Method for antifungal disk diffusion susceptibility testing of yeasts; approved guideline-second edition. 2nd ed. Wayne, PA: CLSI; 2009.

12. Zhang L, Xiao M, Wang H, et al. Yeast identification algorithm based on use of the Vitek MS system selectively supplemented with ribosomal DNA sequencing: proposal of a reference assay for invasive fungal surveillance programs in China. J Clin Microbiol. 2014;52(2):572-577.

13. Canton E, Peman J, Hervas D, et al. Comparison of three statistical methods for establishing tentative wild-type population and epidemiological cutoff values for echinocandins, amphotericin B, flucytosine, and six Candida species as determined by the colorimetric Sensititre YeastOne method. J Clin Microbiol. 2012;50(12):3921-3926.

14. Canton E, Peman J, Iniguez C, et al. Epidemiological cutoff values for fluconazole, itraconazole, posaconazole, and voriconazole for six Candida species as determined by the colorimetric Sensititre YeastOne method. J Clin Microbiol. 2013;51(8):2691-2695.

15. CLSI. M27-S4. Reference method for broth dilution antifungal susceptibility testing of yeasts, fourth informational supplement. 4th ed. Wayne, PA: CLSI; 2012.

16. Castanheira M, Deshpande LM, Davis AP, Rhomberg PR, Pfaller MA. Monitoring antifungal resistance in a global collection of invasive yeasts and molds: application of CLSI epidemiological cutoff values and whole genome sequencing analysis for detection of azole resistance in Candida albicans. Antimicrob Agents Chemother. 2017;61(10). pii: e00906-17.

17. Hernandez S, Lopez-Ribot JL, Najvar LK, McCarthy DI, Bocanegra R, Graybill JR. Caspofungin resistance in Candida albicans: correlating clinical outcome with laboratory susceptibility testing of three isogenic isolates serially obtained from a patient with progressive Candida esophagitis. Antimicrob Agents Chemother. 2004;48(4):1382-1383. 
18. Farmakiotis D, Tarrand JJ, Kontoyiannis DP. Drug-resistant Candida glabrata infection in cancer patients. Emerg Infect Dis. 2014;20(11): 1833-1840.

19. Garcia-Effron G, Kontoyiannis DP, Lewis RE, Perlin DS. Caspofunginresistant Candida tropicalis strains causing breakthrough fungemia in patients at high risk for hematologic malignancies. Antimicrob Agents Chemother. 2008;52(11):4181-4183.
20. Pasquale T, Tomada JR, Ghannoun M, Dipersio J, Bonilla H. Emergence of Candida tropicalis resistant to caspofungin. JAntimicrob Chemother. 2008;61(1):219.

21. Alexander BD, Johnson MD, Pfeiffer CD, et al. Increasing echinocandin resistance in Candida glabrata: clinical failure correlates with presence of $F K S$ mutations and elevated minimum inhibitory concentrations. Clin Infect Dis. 2013;56(12):1724-1732. 


\section{Supplementary material}

Table SI CBPs and ECVs used in the present study

\begin{tabular}{|c|c|c|c|c|c|c|c|c|c|c|}
\hline \multirow[t]{3}{*}{ Antifungal agents } & \multicolumn{5}{|c|}{ Candida tropicalis } & \multicolumn{5}{|c|}{ Candida glabrata } \\
\hline & \multicolumn{4}{|c|}{ CBPs (mg/L) } & \multirow{2}{*}{$\begin{array}{l}\text { ECVs } \\
(\mathrm{mg} / \mathrm{L})\end{array}$} & \multicolumn{4}{|c|}{ CBPs (mg/L) } & \multirow{2}{*}{$\begin{array}{l}\text { ECVs } \\
\text { (mg/L) }\end{array}$} \\
\hline & $\overline{\mathbf{S}}$ & SDD & I & $\mathbf{R}$ & & $\overline{\mathbf{S}}$ & SDD & I & $\mathbf{R}$ & \\
\hline Fluconazole & $\leq 2$ & 4 & - & $\geq 8$ & - & - & $\leq 32$ & - & $\geq 64$ & - \\
\hline Voriconazole & 0.125 & $0.125-0.5$ & - & $\geq 1$ & - & - & - & - & - & 2 \\
\hline Itraconazole & - & - & - & - & I & - & - & - & - & 4 \\
\hline Posaconazole & - & - & - & - & 2 & - & - & - & - & 4 \\
\hline Caspofungin & $\leq 0.25$ & - & 0.5 & $\geq 1$ & - & $\leq 0.12$ & - & 0.25 & $\geq 0.5$ & - \\
\hline Micafungin & $\leq 0.25$ & - & 0.5 & $\geq 1$ & - & $\leq 0.06$ & - & 0.12 & $\geq 0.25$ & - \\
\hline Anidulafungin & $\leq 0.25$ & - & 0.5 & $\geq 1$ & - & $\leq 0.12$ & - & 0.25 & $\geq 0.5$ & - \\
\hline 5-Flucytosine & - & - & - & - & 0.5 & - & - & - & - & 0.25 \\
\hline Amphotericin B & - & - & - & - & 2 & - & - & - & - & 2 \\
\hline
\end{tabular}

Abbreviations: CBP, clinical breakpoint; ECV, epidemiological cut-off value; S, susceptible; SDD, susceptible dose-dependent; I, intermediate; R, resistant.

Infection and Drug Resistance is an international, peer-reviewed openaccess journal that focuses on the optimal treatment of infection (bacterial, fungal and viral) and the development and institution of preventive strategies to minimize the development and spread of resistance. The journal is specifically concerned with the epidemiology of antibiotic

\section{Dovepress}

resistance and the mechanisms of resistance development and diffusion in both hospitals and the community. The manuscript management system is completely online and includes a very quick and fair peerreview system, which is all easy to use. Visit http://www.dovepress.com/ testimonials.php to read real quotes from published authors.

Submit your manuscript here: https://www.dovepress.com/infection-and-drug-resistance-journal 